\title{
Critical Design Issues of Heavy Fuel Turbogas Generators Derived from Automotive Turbochargers. Part II: The Improved Mach Method for off Design Performance Tuning
}

\author{
Luca Piancastelli*, Alessandro Scaltrini, Gian Maria Santi \\ DIN, Università degli Studi di Bologna, Viale Risorgimento, 2, Bologna 40136, Italy
}

Corresponding Author Email: luca.piancastelli@unibo.it

https://doi.org/10.18280/ti-ijes.642-430

Received: 5 January 2020

Accepted: 3 March 2020

\section{Keywords:}

centrifugal compressor, turbogas, automotive turbocharger, improved mach method, off-design performance

\begin{abstract}
The improved Mach method is ideal to evaluate the variations of turboshaft power output with temperature, altitude and humidity. With this original method, the Mach number and the density are evaluated using the equations from acoustics. In this way, simulations are extremely accurate. This paper introduces an off-design example based on a very good turbocharger that reaches a maximum pressure ratio of 5 and is equipped with a high temperature turbine. Automotive turbochargers can be converted to small turbogas generator units. The cost-effectiveness of the solution is furtherly improved by the availability of hybrid turbo-generators with the electronic-converter already included. The design details to overcome the numerous issues of the modification were detailed in the first part of this paper. The resulting unity is a very economical, compact, reliable APU (Auxiliary Power Unit). Unfortunately, the efficiency is around 10\% and the improvement through a recuperator makes the APU not convenient when compared with the ones based on CRDIDs (Common Rail Direct Injection Diesel). In fact, CRDIDs have a best thermodynamic efficiency that is above $50 \%$. The off-design performance of turbochargerderived APUs differs substantially from traditional turboshafts equipped with axial compressors and turbines. Therefore, the performance tuning requires a different approach.
\end{abstract}

\section{INTRODUCTION}

The effects of temperature and pressure altitude are well studied in turbo-machinery. Unfortunately, the centrifugal compressor coupled with the centripetal turbine have their own limitations. The main one is the rotational speed limit. As the temperature and the humidity increase the Mach number, this simple machine tends to accelerate to recover the necessary Mach number ratio. This it is not possible due to limitation in maximum stress. Theoretically, it is possible to improve this limit by changing the material of the compressor impeller and its design. However, the impeller and the diffuser design have been thoroughly studied due to the enormous number of turbochargers installed in vehicles. Impressive improvements have been made in the last 30 years of continuous development, with better design, better material quality and roller bearing units. The turbochargers of this example are the Garrett GTX5533-98 with an additional liner to reduce the impeller blade gap and a turbine made with MAR-M247 TIT that can work up to $1050^{\circ} \mathrm{C}$ continuous temperature. The impeller is polished to improve the maximum pressure ratio obtainable. An advance titanium alloy can also be used instead of the original high-strength aluminum alloy, with limited improvement on maximum rpm, at the price of a much larger cost. The main advantage of the titanium impeller is the capability to withstand higher temperatures that is precious at high altitudes. In the first part of this paper, it was shown that the maximum performance was obtained at about 0.52 the maximum (choke flow) at top rpm. In this condition, the compressor and the turbine have acceptable efficiency while the pressure ratio is nearly maximum. In addition, the compressor $\mathrm{A} / \mathrm{R}$ (Area/Radius) housing sizing is the smaller available to obtain the best pressure/ratio possible. A/R describes the geometry of compressor and turbine housings. It is the discharge (for turbine housings, the inlet) cross-sectional area A divided by the distance $\mathrm{R}$ from the turbo centerline to the centroid of that area. Unfortunately, compressor performance is nearly insensitive to changes in $\mathrm{A} / \mathrm{R}$ (Area/Radius), therefore there are not commercially available $\mathrm{A} / \mathrm{R}$ options available for compressor housings. On the contrary, turbine performance is highly dependent on the $\mathrm{A} / \mathrm{R}$ of the housing, in fact, it affects the maximum flow capacity of the turbine. Smaller A/R, with larger exhaust gas velocity into the turbine wheel, provides quicker boost rise in piston engines, but increases the backpressure and reduces the maximum turbine flow capacity and efficiency. Therefore, in this example, the commercial turbine housing with maximum $\mathrm{A} / \mathrm{R}$ has been chosen for maximum turbogas power.

\section{THE CORRECTED MACH METHOD}

The centrifugal compressor accelerates the flow in the impeller. The degree of reaction of the impeller is limited, therefore the pressure at inlet and outlet of the impeller are similar. The diffuser then decelerates the velocity and transforms it into pressure. In vaneless diffusers, that are very common for the better off-design performance, the flow out of the impeller is supersonic, at high-pressure ratios. The Mach speed is therefore critical for optimum performance. The "Mach speed method" rewrites the traditional mass-flow to 
pressure-ratio maps, by introducing the adimensional parameters $\Psi$ and $\phi(1-2) . \Psi$ and $\phi$ depend on the peripheral tangential speed of the impeller $M u$ expressed with the Mach number. Therefore, the compressor rpm is replaced by the ratio between the peripheral impeller velocity and the sound speed (3). This method is better than the traditional maps that are based on the equivalence of the choke speed. When the compressor encounters thinner air, it naturally tends to increase speed to recover pressure. This fact is "naturally" translated into the "equivalent Mach speed method". The "Mach method" is introduced in paper [1]. The compressor head (total pressure) against inlet air velocity (Mach) for different shaft speeds define a non-dimensional performance (map). The efficiency curves are then drawn on this "invariant" map. Ravi et al. [1] claims a 3\% accuracy. Eqns. (1-3) are the "equivalent Mach speed method" in the International System of Units. In Eqns. (1-3) $R$ is the impeller outer diameter.

$$
\begin{gathered}
\psi=\frac{2 g H}{(\omega R)^{2}} \\
\phi=\frac{Q}{\pi \omega R^{3}} \\
M u=\frac{\omega R}{M}
\end{gathered}
$$

Eq. (3) replaces the rotational speed of the impeller with the relative Mach number $\mathrm{Mu}$ (9). A new set of curves can be drawn at constant $\mathrm{Mu}$ for each curve of $\phi$ and $\Psi$. Then, the Authors improved the method by taking into account of vapor and $\mathrm{CO}_{2}$ content in ambient air by using Eqns. (4) (Table 1) and (5) [2].

$$
\begin{aligned}
& M=a_{0}+a_{1} t+a_{2} t^{2}+\left(a_{3}+a_{4} t+a_{5} t^{2}\right) x_{w} \\
& +\left(a_{6}+a_{7} t+a_{8} t^{2}\right) p_{s}+\left(a_{9}+a_{10} t+a_{11} t^{2}\right) x_{c} \\
& +a_{12} x_{w}^{2}+a_{13} p_{s}^{2}+a_{13} x_{c}^{2}+a_{15} x_{w} p_{s} x_{c}
\end{aligned}
$$

The new total pressure $\rho g H$ can then be calculated (5)

$$
\begin{aligned}
& \rho=\left(3.48349+1.44\left(x_{c}-0.0004\right)\right) \times 10^{-3} \\
& +\frac{p_{s}}{T}\left(1-0.378 x_{w}\right)
\end{aligned}
$$

The advantage of this method is that it takes into account also of the Reynolds number, which, with the sound speed, largely affects the compressor performance.

Table 1. Data of Eq. (4)

\begin{tabular}{cccc}
\hline a0 & 331.5024 & a8 & $2.9310^{-10}$ \\
\hline $\mathrm{a}_{1}$ & 0.603055 & $\mathrm{a}_{9}$ & -85.20931 \\
\hline $\mathrm{a}_{2}$ & -0.000528 & $\mathrm{a}_{10}$ & -0.228525 \\
\hline $\mathrm{a}_{3}$ & 51.471835 & $\mathrm{a}_{11}$ & $5.9110^{-5}$ \\
\hline $\mathrm{a}_{4}$ & 0.1495874 & $\mathrm{a}_{12}$ & -2.835149 \\
\hline $\mathrm{a}_{5}$ & -0.000782 & $\mathrm{a}_{13}$ & $-2.1510^{-13}$ \\
\hline $\mathrm{a}_{6}$ & $-1.8210^{-7}$ & $\mathrm{a}_{14}$ & 29.179762 \\
\hline $\mathrm{a}_{7}$ & $3.7310^{-8}$ & $\mathrm{a}_{15}$ & 0.000486 \\
\hline
\end{tabular}

\section{THE INFLUENCE OF TEMPERATURE ON TURBOGAS PERFORMANCE}

The Mach speed varies with temperature and humidity (Figure 1). The red line is referred to saturated air, while the black one is for dry air. Over 40 DEG C the sound speed varies significantly with saturation. This means that the turbogas will run ad higher speed to achieve the same head; in fact, the compressor works at constant relative speed.

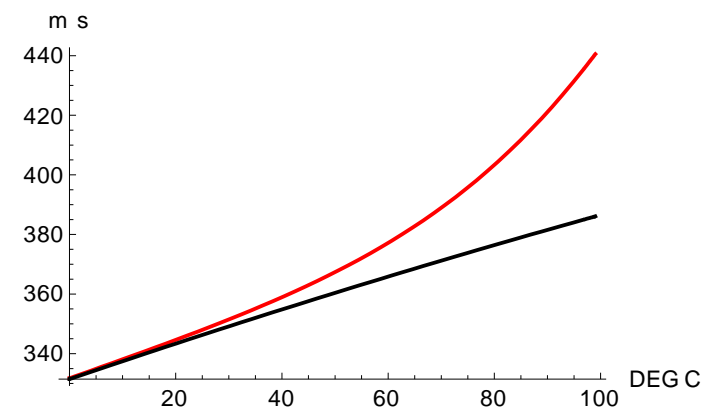

Figure 1. Sound speed [m/s] vs. Temperature [DEG C] sea level. Red=saturated, black=dry air. Cavity geometry

If, as in the case of this turbogas at full power, the speed is already the maximum allowed, it will result in a reduction of the maximum power. Without the problem of condensation, the effects of humidity on gas turbine performance depend on its absolute value, which affects the gas properties of the cycle. Atmospheric elements like humidity, pressure and temperature, shift engine parameters from their dry, nominal values. However, any shift must be considered along with accompanying shifts in other parameters. To derive possible corrections for a shift, certain boundary conditions should be chosen to be fixed. In our case, these boundary conditions are the maximum, allowable constant speed and the maximum turbine wheel temperature $T_{3}$. The nominal, ambient, reference point for this Garrett turbocharger is ISA+33F@2000ft. As it will be shown, it is not possible to make corrections other than shifting slightly the optimum point on the $84,000 \mathrm{rpm}$ curve (of maximum allowed stress/speed) by adjusting the air flow rate. The maximum cycle temperature at turbine inlet is limited to $T_{3}=1050$ DEG C by the turbine material, while the compressor map tends to shrink the allowable-mass-flowrange from surge-to-choke with increasing rpm. The optimum point is always given by the best compromise between mass flow and compression efficiency. Turbine efficiency at high flow rates is nearly constant. Maximum power can be calculated from Eqns. (6-11). The pressure after the compressor $p_{2}$ is given by Eq. (6) way that section titles and other headings are displayed in these instructions, is meant to be followed in your paper.

$$
\begin{gathered}
p_{2}=\left(p_{0}-\Delta p_{\text {filter }}\right) \beta_{c} \\
T_{2}=T_{1}+T_{1}\left(\beta_{c}^{\frac{R}{c_{p}}}-1\right) \cdot \eta_{c}
\end{gathered}
$$

Eqns. (6-9) are for the isentropic to real compression and expansion. The pressure at the intake of the turbine is reduced by the combustion chamber pressure drop (expansion ratio) p_ratiocomb (8). 


$$
\begin{gathered}
\beta_{T}=\frac{p_{-} \text {ratio }_{\text {comb }} p_{2}}{p_{0}+\Delta p_{\text {nozzle }}} \rightarrow T_{4 i s}=T_{3} \cdot \frac{1}{\beta_{T}^{\frac{R}{c_{p f}}}} \\
T_{4}=T_{3}+\left(T_{3}-T_{4 i s}\right) \cdot \eta_{T}
\end{gathered}
$$

From the results of Eq. (10), it is possible to calculate the shaft power output $\mathrm{P}$ to the generator (10). $c_{p f}$ is assumed to be $0.977 c_{p}$ from experimental tests with automotive diesel fuel (Figure 2).

$$
P=\dot{m} \eta_{0}\left(c_{p f}\left(T_{3}-T_{4}\right)-c_{p}\left(T_{2}-T_{1}\right)\right)
$$

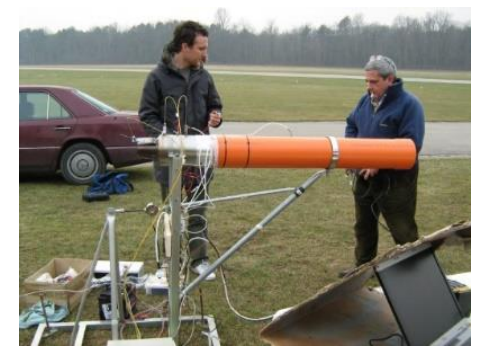

Figure 2. Experimental tests on an early version of the turbogas

The results from equation (10) at reference conditions are shown in Figure 3. The point of maximum power is $204 \mathrm{~kW}$ (a) $52 \%$ flow. The minimum flow ( $\eta \mathrm{c}=0.54$-surge) at 84,000 $\mathrm{rpm}$ is $186 \mathrm{lb} / \mathrm{min}(0 \%)$, while the maximum is $223 \mathrm{lb} / \mathrm{min}$ (choke-100\%). Therefore, 52\% of the flow corresponds to 205 $\mathrm{lb} / \mathrm{min}$. The same map at standard ISA (International Standard Athmosphere), sea-level ambient conditions is shown in Figure 4. However, at the much lower temperature of 15 DEG $\mathrm{C}$ of standard ISA, the maximum rpm for the equivalent Mach Method is $82,000 \mathrm{rpm}$ instead of the $84,000 \mathrm{rpm}$ at Garrett reference. Therefore, using the original (slightly improved) Garrett map, the maximum power at standard ISA ambient conditions is $243 \mathrm{~kW} 982,000 \mathrm{rpm}$. At maximum "material stress" rpm, extrapolating the map, we obtain 259 kW@ $84,000 \mathrm{rpm}$. The Authors do not know whether this later higher value is true, since map extrapolation is physically unsound for a compressor that has a compression ratio around 5. Expensive, climatic chamber tests are necessary to prove this extrapolated value.

Accordingly, the minimum rpm value shifts from 62,000 rpm of Figure 3 down to the 60,500 rpm shown in Figure 4 due to the different Mach number. Figure 5 shows how the ambient temperature has a large influence on maximum power output. Figure 6 shows that even at 40 DEG C, where the saturated vapor content increases significantly the sound speed, the influence of air saturation is limited. The problem of condensing water vapor is however huge and should be treated at air filter level. On the contrary, the influence of altitude is significant (Figure 7). The power loss is almost linear, since the reduction in temperature is partially compensated the reduction in pressure. This fact is shown in Pilot's Flight Manuals when the turbine is not flat rated. In our case, flat rating the turbogas may mean to adapt the maximum temperature $\mathrm{T} 3$ to the worst ambient conditions by limiting the fuel flow. However, this solution gives high penalty on efficiency and it is more convenient to vary the shaft rpm. If it were decided to "flat rate" the turbogas to the maximum value achievable at ISA+25@4,000 m, the maximum power output would be $174 \mathrm{~kW} @ 84,000 \mathrm{rpm}$. Again, this point lies outside the original reference map. If the point should lie inside the reference map, the maximum power output is $155 \mathrm{~kW}$ @ $80,000 \mathrm{rpm}$. In fact, the Mach speed passes from $349 \mathrm{~m} / \mathrm{s}$ at Garrett reference conditions to $333 \mathrm{~m} / \mathrm{s}$ at ISA+25@4,000 m $100 \%$ humidity (4).

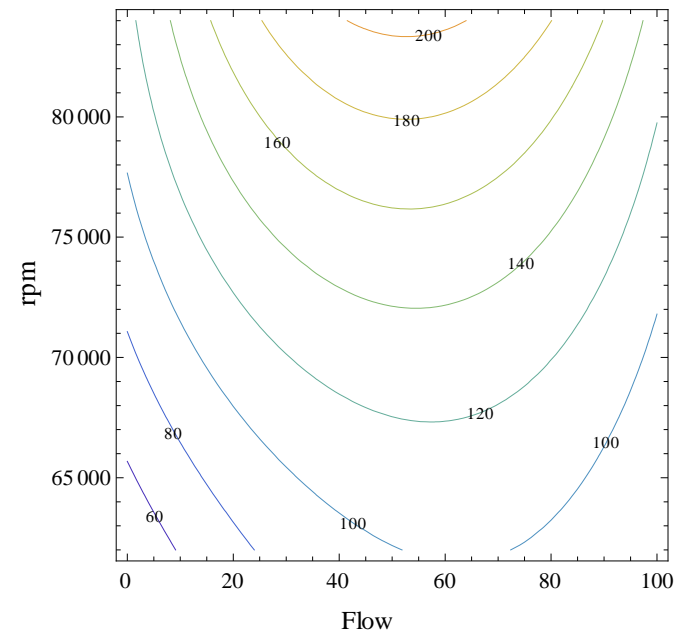

Figure 3. Contour plot of Power [kW] vs. (Flow [\%] - shaft $\mathrm{rpm}$ ) at reference (Garrett) conditions

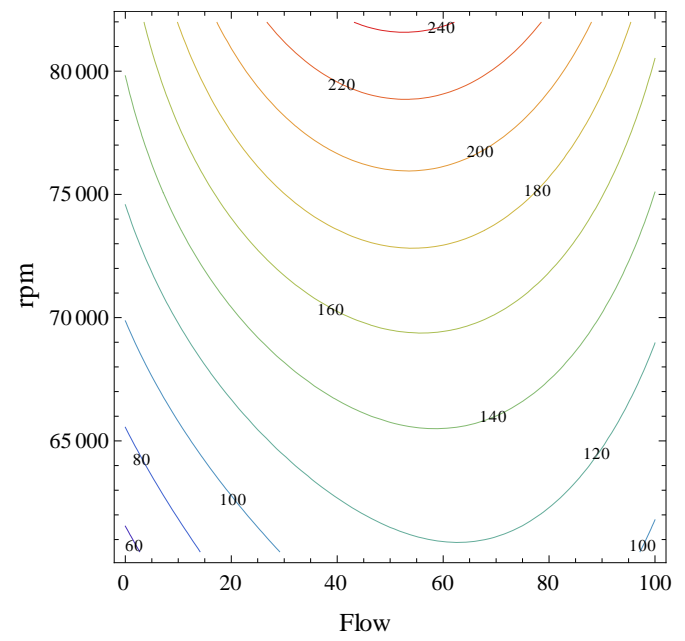

Figure 4. Contour plot of Power [kW] vs. (Flow [\%] - shaft $\mathrm{rpm})$ at ISA standard conditions (sea level)

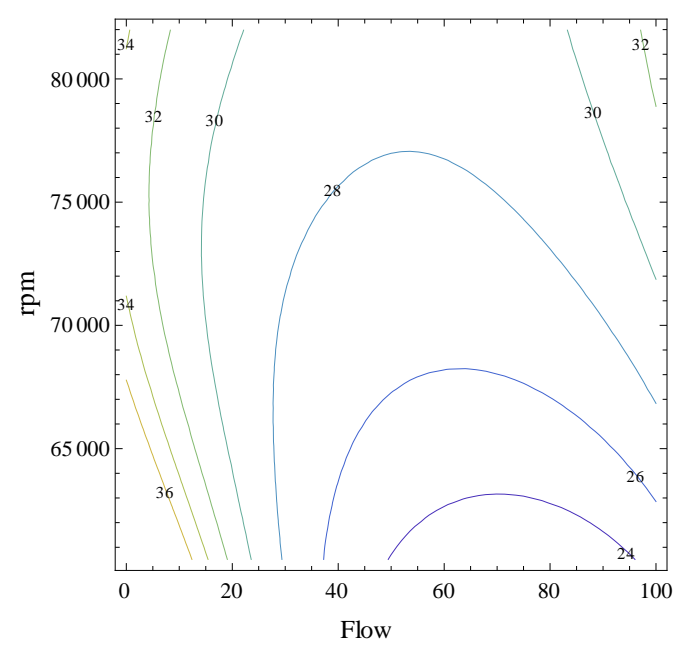

Figure 5. Reduction of $P[\%]$ from 15 DEG-C saturated air to 40 DEG-C-dry vs. (Flow [\%] - shaft rpm) at sea level 


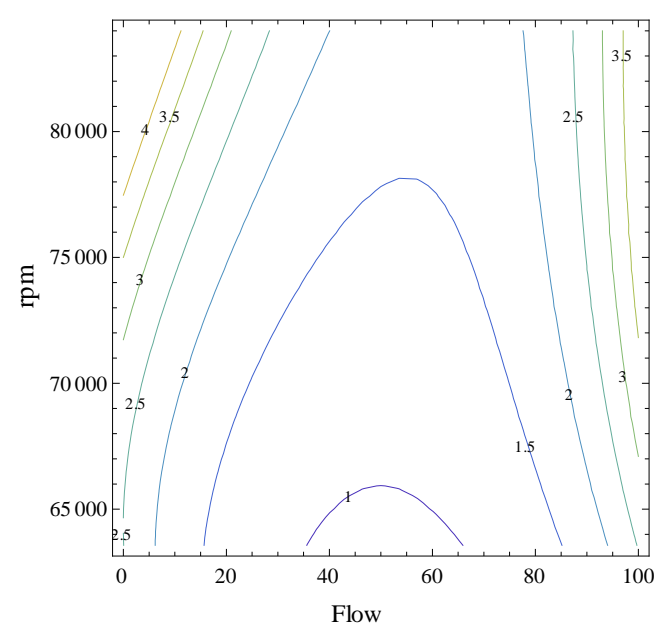

Figure 6. Reduction of $P[\%]$ at 40 DEG-C (sea level) from dry to saturated air vs. (Flow [\%] - shaft rpm)

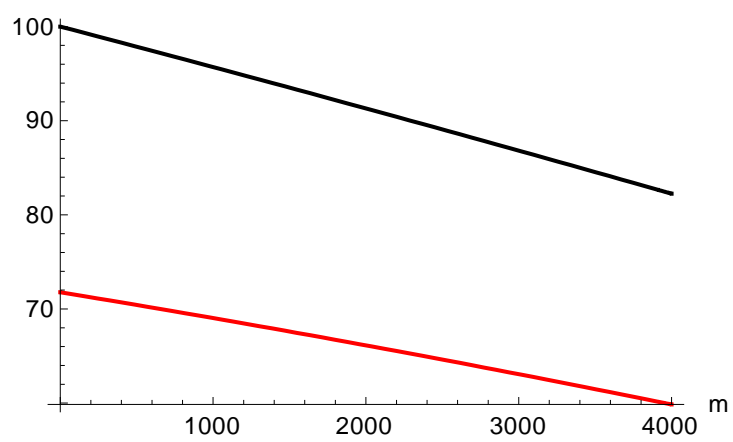

Figure 7. $P[\%]$ with altitude $[\mathrm{m}]$ at ISA (black line) and ISA+25 (red line) - humidity 100\%

Beside the loss of power and efficiency, the decision to flatrate the turbogas generator by reducing the maximum temperature $T_{3}$ may put the compressor in unstable conditions. It is therefore very convenient to vary the turbogas speed. In any case, as pointed out in the first part of this paper, an electronic conversion box is necessary to adapt the output to the electric power grid. The problem is to determine whether it is sufficient to design a suitable generator with the correct power to shaft velocity curve, without further controls on generator torque page.

\section{EFFICIENCY}

In the part 1 of this paper, the possibility of a simple variable-speed permanent-magnet generator in nominal conditions was positively evaluated. The efficiency of the turbogas APU (Auxiliary Power Units) depends on the turbogas true efficiency, the accessories and the electric generation system [3-7].

Therefore, the real efficiency of the APU (Auxiliary Power System) is difficult to evaluate and relatively easy to measure. An approximate theoretical efficiency value will be used in this paper (11). Among the other approximations, it assumes a unitary efficiency of the electric generation system and it does not take into account of the accessories.

$$
\eta=\frac{\dot{m}\left(c_{p f} T_{3}-c_{p} T_{2}\right)}{P}
$$

Figure 8 shows that the compressor has a pretty large high efficiency area. Compressor efficiency influences the whole turbogas efficiency due to the nearly constant turbine efficiency at "feasible shaft rpm". By comparing Figures 3 and 8 , that are both drawn at Garrett-reference-conditions, the flat rate value of $155 \mathrm{~kW}$ lies in the center of the best efficiency area. As altitude, temperatures and humidity vary, the maps move slightly due to the different velocity of sound and it is possible to keep the $52 \%$ flow line to compensate for these variations and to vary the rpm to keep the $155 \mathrm{~kW}$ maximum power value. The problem arises when the minimum power value at acceptable efficiency is required. The turbocharger cannot shift below 30,000 rpm at nominal Garrett reference conditions. The minimum power curve at 52\% (maximum efficiency) with $100 \%$ humidity and $\boldsymbol{T}_{3}=1050$ DEGC are shown in Figure 9.

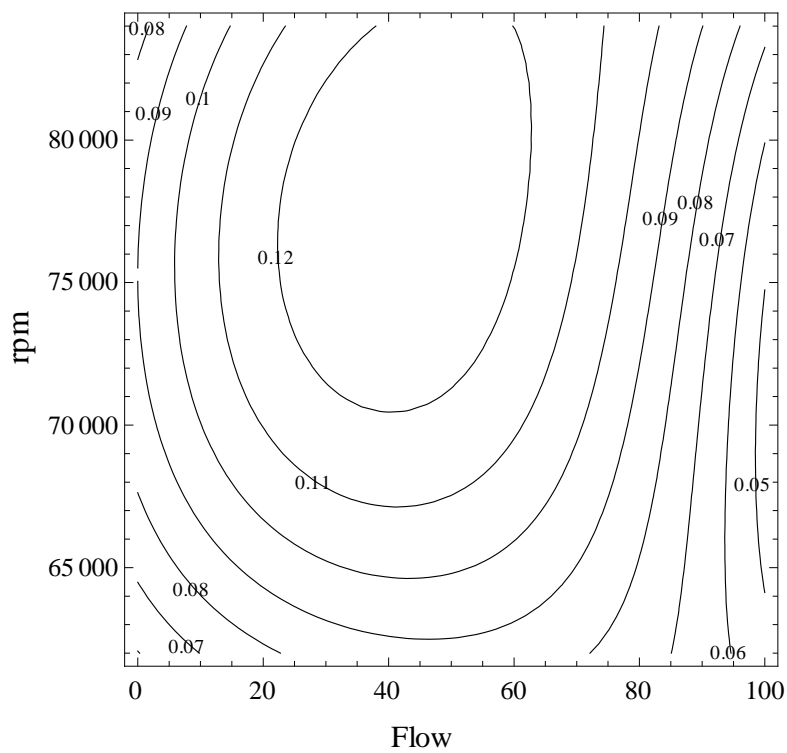

Figure 8. Approximate efficiency (11) vs. (Flow [\%] - shaft $\mathrm{rpm})$ at Garrett reference conditions

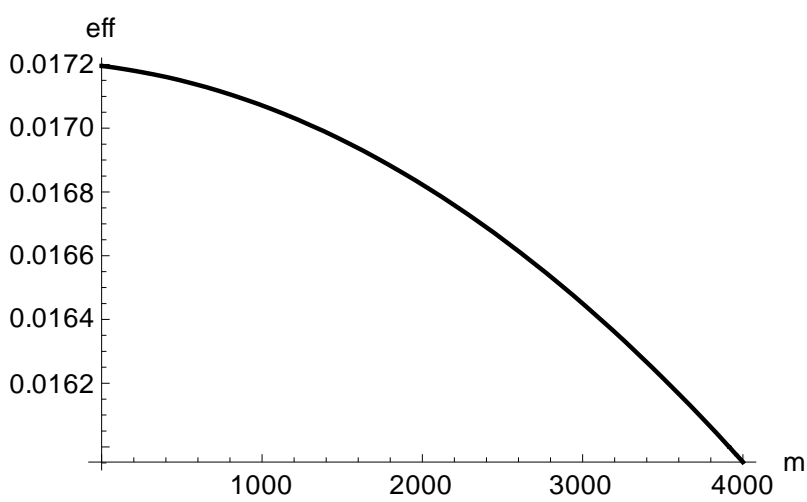

Figure 9. Maximum efficiency (11) at minimum turbogas $\operatorname{rpm}(\sim 30,000 \mathrm{rpm})$ altitude $[\mathrm{m}]-T_{3}=1050 \mathrm{DEG} \mathrm{C}$ and humidity $100 \%$

Figure 9 shows that at minimum rpm the efficiency is very small. Figure 10 shows that also the power is quite small. A reduction in the maximum temperature $T_{3}$ of the cycle affects significantly cycle efficiency. Figure 11 shows the approximate efficiency with $T_{3}=750 \mathrm{DEG} \mathrm{C}$. This value is the maximum allowed with the commercial Garrett turbogas with standard (low cost) turbine. 


\section{CONCLUSIONS}

This paper is divided into two parts. The first one dealt with the issues about the conversion of automotive turbochargers into small turbogas generator units. The cost-effectiveness of the solution is furtherly improved by the recent availability of hybrid turbo-generators ready to use with the electronic converter. The design of these units, especially for heavy fuels, is quite difficult and has numerous issues to be solved, like the combustion chamber design, its interface to the turbocharger unit and the bearing lubrication. The methods to overcome these numerous problems were briefly detailed in the first part of this paper.

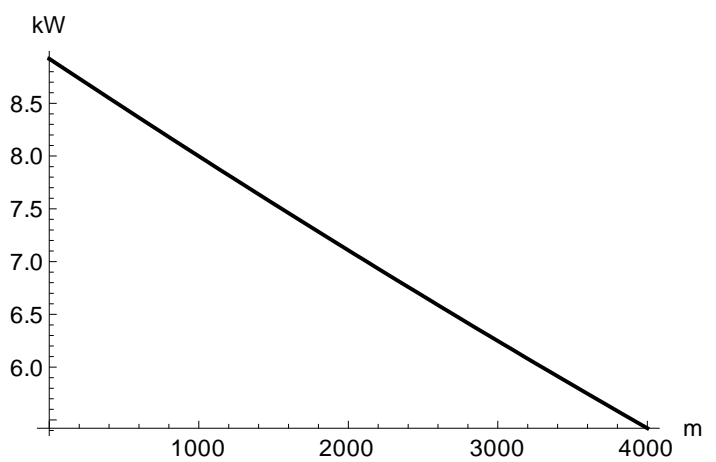

Figure 10. $P[\mathrm{~kW}] @$ minimum turbogas rpm with altitude [m] - T3=1050 DEG C and humidity $100 \%$ at maximum efficiency

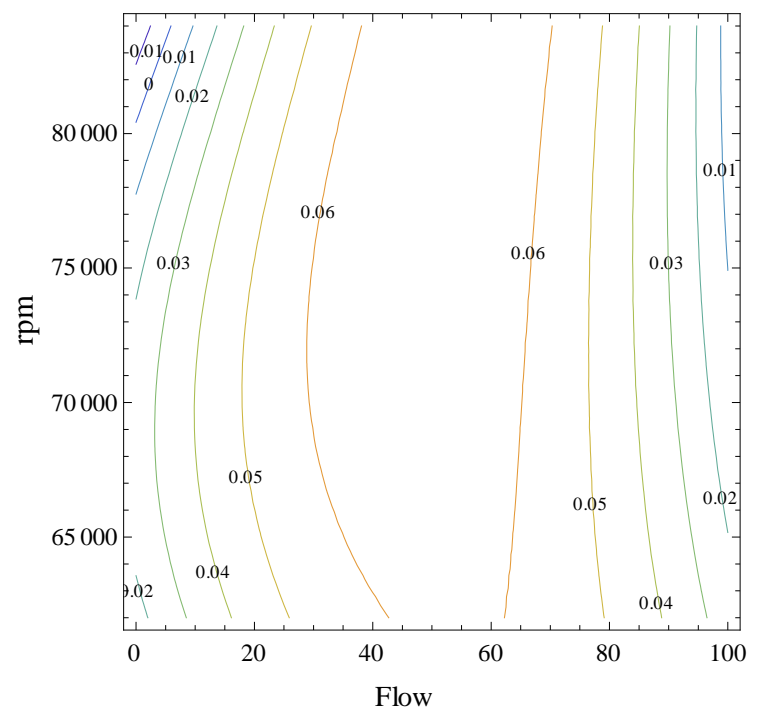

Figure 11. Efficiency (11) with standard turbine wheel $T_{3}=750 \mathrm{C}$ and big turbine housing - standard Garrett reference conditions

The resulting APU (Auxiliary Power Unit) is very economical, compact and reliable. Unfortunately, the fuel consumption is high, especially in out of design conditions. The reference design condition is usually at maximum power output. The best efficiency is around $10 \%$. It can be improved with a recuperator. However, the adoption of the cumbersome recuperator makes the APU not convenient when compared with APUS based on CRDIDs (Common Rail Direct Injection Diesel). In fact, CRDIDs have a better thermodynamic efficiency (well above 50\%) and an impressive off design performance. The CRDID conversion is very easy because the commercial automotive ECUs (Engine Control Unit) have already an embedded PID (Proportional Integral Derivative) control software. Therefore, for CRDIDs, it is sufficient to program the engine at the best rpm, usually between $2,000 \mathrm{rpm}$ and 3,800 rpm, and to assemble an alternator. In most cases, the alternator can be directly "made" on the flywheel. The only true advantages of the turbocharger based APUs are compactness and weight. In fact, the brushless generator runs at the very high speed of the turbochargers, from 80,000 up to $250,000 \mathrm{rpm}$ depending on the size of the turbocharger. This permanent magnet, high-voltage alternator necessitates of an electronic converter down to $48-12 \mathrm{~V} \mathrm{DC}$ for vehicles and the $380 \mathrm{~V}-220 \mathrm{~V} 50 \mathrm{~Hz}-60 \mathrm{~Hz}$ for stationary installation. Another important issue of these turbogas units is the control and optimization of off-design performance. For this purpose, the improved Mach method proposed by the Authors is ideal to evaluate the variation of power output with temperature, altitude and humidity. In fact, with the "improved Mach number method", the velocity of sound and density are evaluated using the equations from acoustics. In this way, turbogas simulations are extremely easy and accurate. They also include the effect of temperature, pressure, humidity and $\mathrm{CO}_{2}$. To describe this concept, this paper introduces an example based on a very good, very large turbocharger that reaches a maximum pressure ratio of 5 and has a high temperature turbine. Large turbochargers have the best efficiency due to the reduced gap-to-size and the better Reynolds numbers. The modified Mach method is based on the concept that the centrifugal compressor works with constant Mach number and not with rpm-chock-limit as in the traditional approach. In this way, the off-design evaluation of the compressor is very accurate. On the other side, the turbine works usually at maximum efficiency, being a much "easier" machine. Therefore, an accurate simulation of the compressor is fundamental for the turbogas tuning. The very simple turbogas APU of this example is composed by a standard turbogas in the best commercially available version. This means the best turbine available for $\mathrm{A} / \mathrm{R}$ housing and material (maximum temperature 1050 DEG $\mathrm{C}$ ). This turbocharger is a very large unit for automotive use. Therefore, it reaches the topmost values in term of performance and efficiency. Still, the best overall efficiency is around $10 \%$ for the turboshaft. The maximum power output is around $250 \mathrm{~kW}$ at sea level ISA. In order to have a simple control system with a high voltage permanent magnet generator coupled to the shaft, the best regulation technique is to flat rate the unit to the maximum available power at worst conditions $(4,000 \mathrm{~m}-\mathrm{ISA}+25-100 \%$ humidity). This means that at ground level the turboshaft will run at about $70,000 \mathrm{rpm}$ at Garrett reference ambient conditions (ISA+33 F @ $2000 \mathrm{ft}$ ). The maximum fuel flow set by the control unit will keep the turbogas in these conditions at full load. The "right" rpm and airflow will be found automatically by the turbogas. The control unit will check not to pass the maximum temperature allowed of 1,050 DEG C at turbine inlet. The torque required from the generator at full power will determine this equilibrium point. As temperature, humidity and altitude increases, the turbogas unit will automatically increase the rpm to elaborate a sufficient air mass. The working point would remain on the curve of maximum efficiency up to the topmost speed of $84,000 \mathrm{rpm}$. If this speed can be reached by the turbogas is not clear since, in many conditions, the compressor is out of map. However, being a commercial unit, it probably can reach the topmost speed with reduced efficiency. In our flat rated example, 
overspeed occurs only when altitude or temperature exceed the combination of $4,000 \mathrm{~m}$ and ISA +25 degrees (the ISA curve with 40 DEG $\mathrm{C}$ at sea level). The control system of the turbogas will therefore be quite simple with a "conditioner" on the electric power output. The "conditioning unit" is necessary in any case to adjust the electric parameters to national grids and vehicles. This turbogas is compact $(\sim \mathrm{W}=400 \mathrm{~mm}$, $\mathrm{H}=600 \mathrm{~mm}, \mathrm{~L}=500 \mathrm{~m})$, lightweight $(\sim 50 \mathrm{~kg})$ and cost-effective. The best efficiency will be below $10 \%$ at full power. At the lowest power output, the efficiency will be extremely low. For this reason, the use with a battery is convenient in case of high load variations. The capability to work with most fuels (LPG, $\mathrm{NG}$, jet $\mathrm{A} 1$, diesel, gasoline) renders the units extremely versatile. The installation is also quite simple since the unit is air cooled. Unfortunately, there are many shortcomings. The air filter should ingest high flow rates of air and dirt. Therefore, filter cleaning is problematic in dirty and sandy environments with cleaning intervals that can be on daily basis. The filter would be quite large as it can be seen in vehicle installations like the Abrams tank. Another installation problem is the high exhaust temperature ( $~ 800$ DEG $C$ ) with the possibility of flames on starting. Idling is also a shortcoming, with fuel consumption that can be $40 \%$ the one at full power.

\section{REFERENCES}

[1] Ravi, A., Sznajder, L., Bennett, I. (2015). Compressor map prediction tool. 9th International Conference on Compressors and their Systems. IOP Conf. Series: Materials Science and Engineering, 90: 012042. https://doi.org/10.1088/1757-899X/90/1/012042

[2] Rasmussen, K. (1997). Calculation methods for the physical properties of air used in the calibration of microphones. DEP. of Acoustic Technology Technical University of Denmark Report PL-11b, 20p. DTU, DTULibrary. on line, https://orbit.dtu.dk/en/publications/calculation-methodsfor-the-physical-properties-of-air-used-in-th.

[3] Piancastelli, L., Burnelli, A., Cassani, S. (2017). Validation of a simplified method for the evaluation of pressure and temperature on a RR Merlin XX head. International Journal of Heat and Technology, 35(1): 549-558. https://doi.org/10.18280/ijht.350311

[4] Piancastelli, L. (2019). Domestic micro-cogeneration: A high efficiency, cost effective, simple solution. TECNICA ITALIANA-Italian Journal of Engineering Science, 63(1): 46-51. https://doi.org/10.18280/tiijes.63010

[5] Piancastelli, L., Baldassarri, A., Pezzuti, E. (2020). On the energy management of the new formula 1 powerplants. TECNICA ITALIANA-Italian Journal of Engineering $\quad$ Science, 64(1): 95-102. https://doi.org/10.18280/ti-ijes.640115

[6] Piancastelli, L., Peli, F. (2018). The advantage of the split turbocharger in Formula 1 engines. TECNICA ITALIANA-Italian Journal of Engineering Science, 61+1(1): 36-41. https://doi.org/10.18280/ti-ijes.620105

[7] Shaik M.I., Fazle, M., Prathi, V.K., Lorenzini, G.,
Lorenzini, E. (2018). Cattaneo-Christov heat flux on UCM flow across a melting surface with cross diffusion and double stratification. TECNICA ITALIANA-Italian Journal of Engineering Science, 61+1(1): 12-21. https://doi.org/10.18280/ti-ijes.620102

\section{NOMENCLATURE}

Description

Value

g

Gravitational acceleration $\left[\mathrm{m} \mathrm{s}^{-2}\right] 9.81$

Head [m]

Shaft angular velocity $\left[\mathrm{s}^{-1}\right]$

Impeller radius $[\mathrm{m}]$

0.0665

Flow rate inlet $\left[\mathrm{m}^{3} \mathrm{~s}^{-1}\right]$

Adimensional parameter "mass

flow" for compressor map

$\Phi$

Adimensional parameter "head" for compressor map

$\mathrm{Mu} \quad$ Adimensional parameter "speed" for compressor map

Sound velocity inlet $\left[\mathrm{m} \mathrm{s}^{-1}\right]$

Inlet temperature [DEG C]

Mole fraction of water vapor in air

Mole fraction of $\mathrm{CO}_{2}$ in air

0.0004

$\mathrm{x}_{\mathrm{W}}$

Inlet static pressure [Pa]

Inlet air density $\left[\mathrm{kg} \mathrm{s}^{-3}\right]$

Ambient static pressure [bar]

Filter pressure drop [bar]

Compressor pressure ratio

Ideal gas constant $\left[\mathrm{kPa} \mathrm{m}^{3}(\mathrm{~kg} \mathrm{~K})\right.$ 1]

$c_{p} \quad$ Specific heat of air at constant pressure $\left[\mathrm{kJ}(\mathrm{kg} \mathrm{K})^{-1}\right]$

$\mathrm{T}_{1} \quad$ Temperature compressor inlet [K]

$\mathrm{T}_{2} \quad$ Temperature compressor outlet [K]

$\mathrm{T}_{3} \quad$ Temperature turbine inlet $[\mathrm{K}]$

$\mathrm{T}_{4} \quad$ Temperature turbine outlet $[\mathrm{K}]$

$\mathrm{c}_{\mathrm{pf}} \quad$ Specific heat of exhaust at constant pressure $\left[\mathrm{kJ}(\mathrm{kg} \mathrm{K})^{-1}\right]$

$\mathrm{T}_{4 \text { is }} \quad$ Temperature turbine outlet isentropic $[\mathrm{K}]$

$\eta_{\mathrm{c}} \quad$ Compressor efficiency

p_ratio ${ }_{\text {comb }}$ Combustor pressure ratio

$\beta_{\mathrm{T}} \quad$ Turbine pressure ratio

$\Delta \mathrm{p}_{\text {nozzle }} \quad$ Nozzle pressure drop [bar] $\quad 0.0135$

$\eta_{\mathrm{T}} \quad$ Turbine efficiency

m' Mass flow rate $\left[\mathrm{kg} \mathrm{s}^{-1}\right]$

$\eta_{0} \quad$ Mechanical efficiency 